\title{
Image-Based Method for In-Vivo Freehand Ultrasound Calibration
}

\author{
Wolfgang Wein and Ali Khamene \\ Imaging \& Visualization Department \\ Siemens Corporate Research, Princeton NJ USA \\ Email: \{wolfgang.wein,ali.khamene\}@siemens.com
}

\begin{abstract}
For freehand ultrasound systems, a calibration method is necessary to locate the position and orientation of a 2D B-mode ultrasound image plane with respect to a position sensor attached to the transducer. In addition, the acquisition time discrepancy between the position measurements and the image frames has the be computed. We developed a new method that adresses both of these problems, based on the fact that a freehand ultrasound system establishes consistent 3D data of an arbitrary object. Two angulated sweeps of any object containing well visible structures are recorded, each at a different orientation. A non-linear optimization strategy maximizes the similarity of 2D ultrasound images from one sweep to reconstructions computed from the other sweep. No designated phantom is required for this calibration. The process can be performed in vivo on the patient. We evaluated our method using freehand acquisitions on both a phantom and the human liver. The accuracy of the approach was validated using a 3D ultrasound probe as a known reference geometry.
\end{abstract}

Keywords: 3D Freehand Ultrasound, Calibration, Reconstruction

\section{INTRODUCTION}

Due to its non-invasive nature, flexibility and cost-effectiveness, medical 2D B-mode ultrasound is a very popular imaging method. It essentially depicts the ultrasonic reflections of human tissue within a two-dimensional crosssection. While 3D ultrasound transducers are now available by most of the manufacturers, tracked freehand ultrasound is nevertheless a very important technology. One one hand, it allows for having larger field-of-view required for acquisitions of whole organs. On the other hand, it is a preferred modality for interventional navigation applications, as both the ultrasound images and their location in space are acquired in real-time.

For such 3D freehand ultrasound systems it is crucial that the spatial relation between the position sensor and the image plane is precisely determined. This is a rather complex problem, which tends to be underrated. A large body of literature deals with spatial ultrasound calibration, a concise survey is. ${ }^{1}$ Most of the methods are based on imaging a designated phantom object with some known geometric properties. Acquiring a number of images from different orientations then allows to establish the relation of the image content to the tracking sensor's coordinate system. Optimal phantoms contain material mimicking human tissue, and are precisely manufactured according to a geometric model used for the mathematical computation of the calibration parameters. Such phantoms are mostly used for commercial freehand ultrasound systems, and potentially allow for a very convenient calibration workflow, that can be performed by sonographers \& physicians. However, purchasing or manufacturing them is often not an option for research facilities. On the contrary, the Single-Wall calibration method ${ }^{2}$ only relies on a rough-textured plane submerged in water. A number of subsequent research effort has been conducted to further automate this method. ${ }^{3}$ However, its precision is still limited by the narrow range of imaging orientations and depths. The varying elevational beam thickness causes the Single Wall phantom to appear as line with different thickness and fuzziness, depending on the depth and focus settings used. Another problem arises as the speed of sound in water is different than the reference speed assumed by ultrasound machines $(1540 \mathrm{~m} / \mathrm{s}$ in average human soft tissue). An additional source of errors is introduced when trying to overcome this problem, either by using a particular fluid with the desired speed (mixture of water with $\mathrm{NaCl}$ ), or estimating the actual speed of sound and henceforth compensating for the image distortion. Phantom-less calibration methods ${ }^{4,5}$ use the intersections of a tracked tool with the ultrasound image plane to derive the calibration. They suffer from the speed-of-sound problem as well, as the calibration has to be performed underwater. Besides, two more error

Medical Imaging 2008: Ultrasonic Imaging and Signal Processing, edited by Stephen A. McAleavey, Jan D'hooge, Proc. of SPIE Vol. 6920, 69200K, (2008) · 1605-7422/08/\$18 $\cdot$ doi: 10.1117/12.769948 


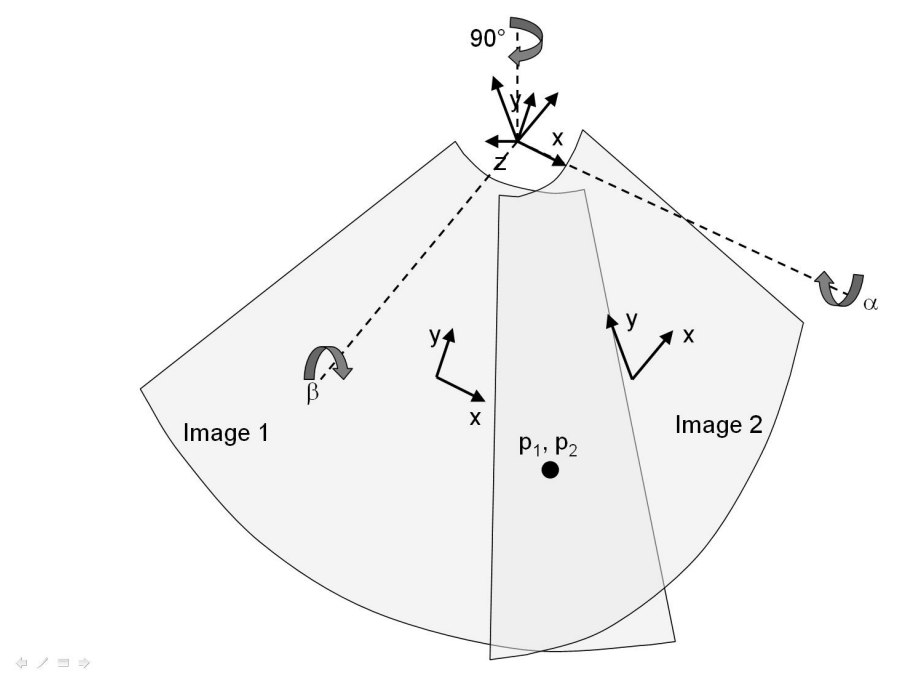

Figure 1. Used coordinate systems and image planes for computing reconstruction errors.

sources are added: The tracking accuracy of the tool itself, as well as the calibration of the tool. For magnetic tracking, especially the rotational accuracy is rather poor, making it difficult to achieve a good tool calibration. For optical tracking, the target markers have to be considerably far away from the tool tip, as they always need to be above the water surface.

In, ${ }^{6}$ an image-based approach is used, which maximizes Mutual Information of tracked ultrasound images and reconstructions from an MRI scan of a custom-built Agar gel phantom.

In our approach, we can avoid a number of the problems pointed out above, by using spatial consistency of realistic freehand ultrasound acquisition as the underlying information for calibration. The proposed method can be performed using in vivo tissue, making it possible to calibrate ultrasound prior to the procedure, or even validate the calibration during an exam. While in ${ }^{7}$ tracking of in-plane motion on successive frames is used to monitor some calibration parameters, we are using large-scale consistency of the anatomy to recover all parameters, including the temporal lag.

\section{METHOD}

If two slow angulated freehand ultrasound sweeps are acquired from the same anatomy at approximately perpendicular orientation, they measure roughly the same image intensities at every point in 3D-space, assuming the right calibration parameters $T_{c}$ are known. While this is not entirely true for small structures, speckle patterns and tissue interfaces yielding specular reflections (i.e. bone) due to the orientation-dependency of ultrasound imaging, it is sufficiently given for large-scale soft tissue structures such as e.g. encountered in liver imaging. Hence a maximization of the similarity measure $S$ of images $I_{j}$ from the first sweep with reconstructions $\widetilde{I}_{j}$ from the second sweep (and vice versa) should yield the correct optimization parameters:

$$
\widetilde{T}_{c}=\arg \max _{T_{c}} \sum_{j} S\left(I_{j}(\vec{p}), \widetilde{I}_{j}(\vec{p})\right)
$$

\subsection{Geometric Formulation}

In homogenous coordinates, we denote the sought calibration $T_{c}$ as rigid transformation from image to sensor coordinates, and $T_{i j}=T_{j}^{-1} T_{i}$ the relative transformation between two sensor measurements. Then the transformation between two images is described as

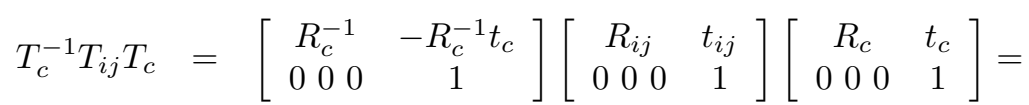




$$
\begin{aligned}
& =\left[\begin{array}{cc}
R_{c}^{-1} & -R_{c}^{-1} t_{c} \\
000 & 1
\end{array}\right]\left[\begin{array}{cc}
R_{i j} R_{c} & R_{i j} t_{c}+t_{i j} \\
00 & 0
\end{array}\right]= \\
& =\left[\begin{array}{cc}
R_{c}^{-1} R_{i j} R_{c} & R_{c}^{-1}\left(R_{i j} t_{c}+t_{i j}-t_{c}\right) \\
000 & 1
\end{array}\right]
\end{aligned}
$$

If the rotation $R_{i j}$ is close to identity, the influence of both $R_{c}$ and $t_{c}$ in equation 2 diminishes, which means that frames used for calibration should be angulated as far as possible from each other. For further examination, we use a more specific setup with $T_{12}=T_{2}^{-1} T_{1}$ and two locations $\overrightarrow{p_{1}}=\left(u_{1}, v_{1}, 0,1\right)^{T}$ and $\overrightarrow{p_{2}}=\left(u_{2}, v_{2}, 0,1\right)^{T}$ in image coordinate systems that represent the same point $\overrightarrow{p_{w}}$ in $3 \mathrm{D}$-space:

$$
T_{c}^{-1} T_{2}^{-1} T_{1} T_{c} \overrightarrow{p_{1}}=\overrightarrow{p_{2}}
$$

Here $T_{c}$ describes the rigid $4 x 4$ transformation matrix of the correct calibration, which maps the ultrasound image coordinate system onto the coordinate system of the tracking sensor. $T_{1}$ and $T_{2}$ are the tracking matrices of images 1 and 2, which describe the transformation from sensor to world coordinate system of the tracking system. The image locations $\overrightarrow{p_{1}}$ and $\overrightarrow{p_{2}}$ are in physical units $(\mathrm{mm})$, i.e. we assume the correct pixel spacing to be known. We are now interested in the reconstruction error $e$ of the 3D positions, if an error-prone calibration matrix $\widetilde{T}_{c}$ is used.

$$
\begin{aligned}
e & =\left|T_{2} \widetilde{T}_{c} \overrightarrow{p_{2}}-T_{1} \widetilde{T_{c}} \overrightarrow{p_{1}}\right| \\
\text { Using eq. 3: } e & =\left|T_{2} \widetilde{T}_{c} T_{c}^{-1} T_{2}^{-1} T_{1} T_{c} \overrightarrow{p_{1}}-T_{1} \widetilde{T_{c}} \overrightarrow{p_{1}}\right|
\end{aligned}
$$

In the setup used to analytically derive the error, $T_{c}$ consists solely of a translation $d=100 \mathrm{~mm}$ along the negative $\mathrm{y}$-axis, $T_{1}$ a rotation of $\alpha$ around the $\mathrm{x}$-axis, $T_{2}$ a rotation of $\beta$ around the $\mathrm{x}$-axis first and $90^{\circ}$ around the $\mathrm{y}$-axis (see figure 1). Without loss of generality, we define $v_{1}=0 \mathrm{~mm}$ and $\alpha=-30^{\circ}$. The $3 \mathrm{D}$ point $\overrightarrow{p_{w}}$ is

$$
\overrightarrow{p_{w}}=T_{1} T_{c} \overrightarrow{p_{1}}=\left(\begin{array}{c}
u_{1} \\
(v-d) \cos \alpha \\
(v-d) \sin \alpha \\
1
\end{array}\right)
$$

The angle $\beta$ is defined such that the second imaging plane intersects $\overrightarrow{p_{w}}$ as well:

$$
\beta=-\arctan \frac{-u_{1}}{(v-d) \cos \alpha}
$$

An error-prone calibration $\widetilde{T}_{c}$ is defined as a translation about $\left(t_{x}, t_{y}-d, t_{z}\right)^{T}$. Inserting in equation 5 yields

$$
e=\left\|\left(\begin{array}{c}
\sin (\beta) t_{y}+\cos (\beta) t_{z}-t_{x} \\
\cos (\beta) t_{y}-\sin (\beta) t_{z}-\cos (\alpha) t_{y}+\sin (\alpha) t_{z} \\
-t_{x}-\sin (\alpha) t_{y}-\cos (\alpha) t_{z} \\
0
\end{array}\right)\right\|
$$

For applying error in one translation component at a time, the error results to:

$$
\begin{aligned}
& e_{x}=\left|t_{x}\right| \sqrt{2} \\
& e_{y}=\left|t_{y}\right| \sqrt{2(1-\cos \alpha \cos \beta)} \\
& e_{z}=\left|t_{z}\right| \sqrt{2(1-\sin \alpha \sin \beta)}
\end{aligned}
$$

The reconstruction error linearly increases with respect to the calibration error, and is independent of the position in the image plane (apart from the dependency of the angle $\beta$ in equation 7). Only if the angles $\alpha$ and $\beta$ are small, the error in the $y$ direction remains unchanged (eq. 10). This is obvious as in that case the change of $t_{y}$ moves the two image planes along parallel lines in 3D space. Hence the calibration sweeps have to be sufficiently angulated, two linear motions would not recover the $y$ translation of the calibration transformation. Similarly, 


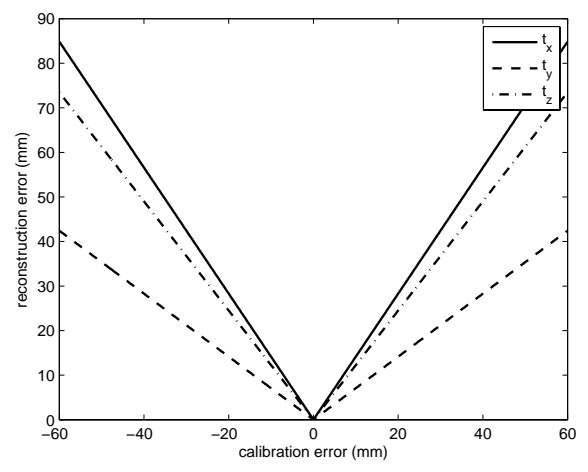

(a) translation error

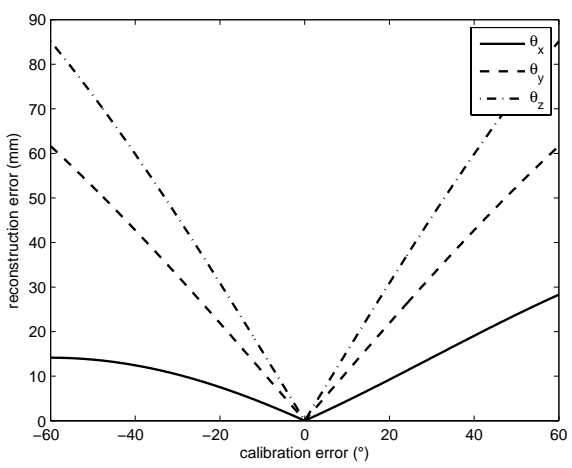

(b) rotation error

Figure 2. Plots of the reconstruction error. The configuration is $\overrightarrow{p_{1}}=(40,20,0,1)^{T}$ and $\alpha=\beta=-30^{\circ}$.

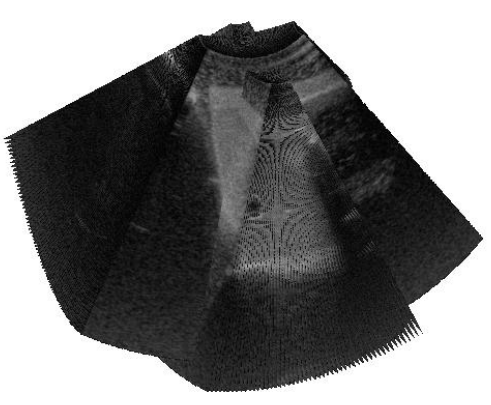

(a) $3 \mathrm{D}$ view

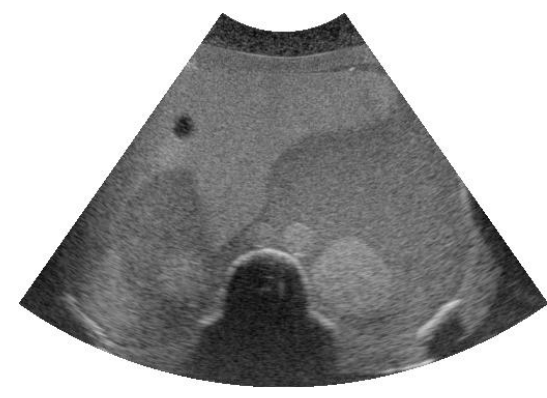

(b) ultrasound image

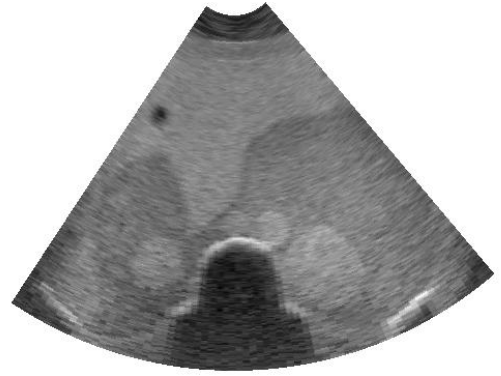

(c) reconstruction

Figure 3. Phantom images, acquired with the Antares wobbler. The NCC measure for those images is 0.86.

eq. 11 shows that for angles approaching $90^{\circ}$ a change in $t_{z}$ would not affect the reconstruction error. However, such a configuration is not possible with transcutaneous ultrasound anyway (the image planes would be parallel to the patient's skin).

Expanding the equations with rotations in $\widetilde{T}_{c}$ yields very large trigonometric equations. Essentially, the only situations where a wrong rotation would not increase the reconstruction error, are: a) that $\overrightarrow{p_{1}}$ and $\overrightarrow{p_{2}}$ both lie on the $\mathrm{x}$ - or $\mathrm{y}$-axis of the respective image coordinate system (which would only be the case for a minority of the pixels in an ultrasound image); b) both $\alpha$ and $\beta$ are very small, then the $\theta_{y}$ rotation cannot be recovered. Figure 2 displays the reconstruction error for all 6 calibration parameters.

We have shown that the described setup allows to recover all 6 calibration parameters. If an appropriate image similarity measure between original ultrasound intensities $I_{i m}(u, v)$ and a reconstruction at its 3D location $I_{r e c}\left(\overrightarrow{p_{w}}\right)$ from the respective other sweep can be described as a monotonous function of the reciprocal reconstruction error $e$, its maximization will result in the correct calibration.

\subsection{Algorithm}

We have previously presented an algorithm to efficiently reconstruct arbitrary planes directly from a freehand ultrasound sweep, ${ }^{8}$ without creating a cartesian reconstruction volume. 5-10 images from every sweep are compared against reconstructions from the other sweep, respectively. Normalized Cross-Correlation (NCC) is computed on every pair, its average is used as a cost function for non-linear optimization (i.e. $S=N C C$ in equation 1). This assures that brightness and contrast differences of an ultrasound image and its reconstruction do not impose the registration accuracy. Such differences can occur, as the respective intensities originate from different scan orientations. The Amoeba Simplex algorithm ${ }^{9}$ then finds the optimal calibration transformation maximizing the image similarity. Instead of directly modifying the calibration parameters, a relative calibration 

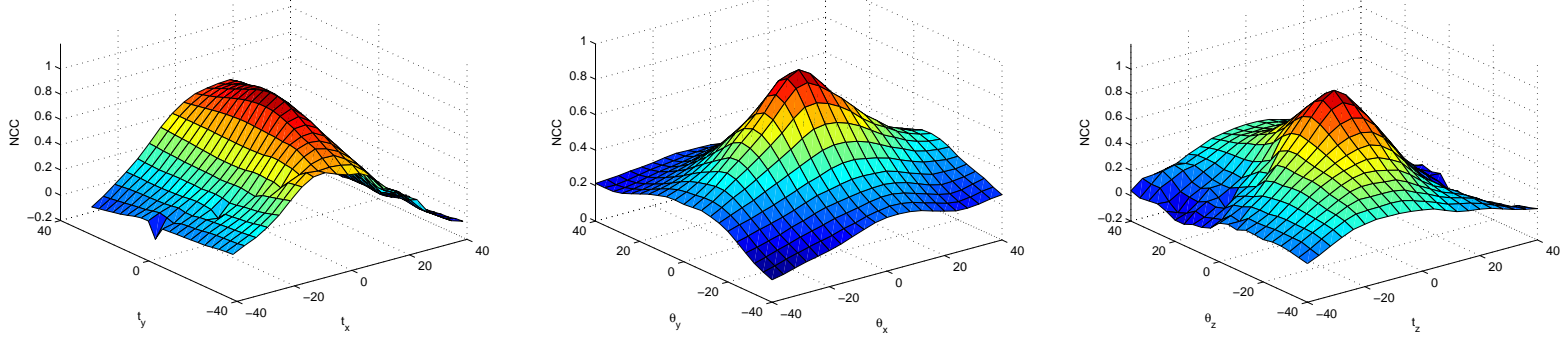

Figure 4. Similarity measure plots for the Antares phantom data, altering two calibration parameters at once.

\begin{tabular}{|c|c|c|c|c|c|c|c|c|c|}
\hline & $t_{x}(\mathrm{~mm})$ & $t_{y}(\mathrm{~mm})$ & $t_{z}(\mathrm{~mm})$ & $\theta_{x}\left({ }^{\circ}\right)$ & $\theta_{y}\left({ }^{\circ}\right)$ & $\theta_{z}\left({ }^{\circ}\right)$ & $\mathrm{NCC}$ & time $(\mathrm{s})$ & error $(\mathrm{mm})$ \\
\hline \multicolumn{10}{|c|}{ Antares Ground Truth data } \\
\hline mean & -0.15 & 2.01 & -0.63 & -0.06 & 0.00 & 0.01 & 0.91 & 168.6 & 2.39 \\
\hline$\sigma$ & 0.20 & 0.75 & 0.30 & 0.38 & 0.17 & 0.15 & 0.00 & 45.9 & 0.74 \\
\hline \multicolumn{10}{|c|}{ Sequoia freehand ultrasound, phantom vs. in-vivo } \\
\hline phantom & 115.5 & -3.5 & 27.3 & -172.7 & 7.3 & -90.3 & 0.85 & 115.9 & \\
\hline liver & 114.4 & -2.0 & 25.8 & -172.8 & 6.8 & -86.6 & 0.68 & 89.2 & \\
\hline
\end{tabular}

Table 1. Results of precision and robustness study.

matrix composed from zero-initialized translation and Euler angles is right-multiplied onto the initial estimate. This avoids the inherent Gimbal lock problem of the Euler-angles parameterization.

\section{RESULTS}

\subsection{Ground Truth Study}

To derive the absolute precision of our new calibration method, we used a Siemens Antares ultrasound machine with the C5F1 3D transducer. It acquires 30-70 2D frames per volume, mechanically wobbling over an angulation of $35-75^{\circ}$. Two perpendicular volumes have been acquired from a multimodal abdominal phantom (CIRS Inc, Norfolk VA USA, figure 3(a)). Using the imaging geometry from the saved pre-scan-converted DICOM volumes, we perform a 2D scan-conversion into a set of cartesian 2D images, and tag the images with calibration and tracking matrices to resemble a freehand ultrasound acquisition. The tracking effectively consists of an x-rotation angle $\alpha$, the calibration of a negative y offset $d$, similarly to the setup in figure 1. A standard imagebased registration technique is used to register 3D-scan-converted representations of the volumes, using NCC as similarity metric. The registration result is then applied to the tracking matrices of the second sweep. Table 1 (top) depicts the mean and standard deviation values for execution of 183 optimizations, each randomly displaced from the ground truth calibration (every parameter in $\pm 20 \mathrm{~mm}, \pm 20^{\circ}$ mean distribution). The method reliably finds the same calibration. Only in the y axis is a systematic bias of $2 \mathrm{~mm}$. This might be due to interpolation in the 2D- and 3D-scan-conversion algorithms, or a difference between the 3D imaging geometry provided by the manufacturer, and the physical transducer properties. The error value in the right column depicts the relocation error of a point in the center of the ultrasound image (around $7 \mathrm{~cm}$ depth). It is mostly composed from that deviation in $t_{y}$. Figure 4 depicts $2 \mathrm{D}$ plots of the sum-of-NCCs similarity function used, for changing two parameters from the ground truth calibration each.

\subsection{Calibration on Human Liver}

Here we used a freehand ultrasound system based on a Siemens Sequoia ultrasound machine in conjunction with an Ascension 3D Guidance magnetic tracking system and a $\mathrm{PC}$ with a video grabber. Three transducers (4C1, 4V1 and 6C2) were calibrated for a permanent clinical setup, using the same CIRS phantom. For every transducer, two perpendicular sweeps were recorded at $12 \mathrm{~cm}, 18 \mathrm{~cm}$ and $26 \mathrm{~cm}$ depth setting, and two focal zones (the minimum on that machine) evenly distributed over the image. 


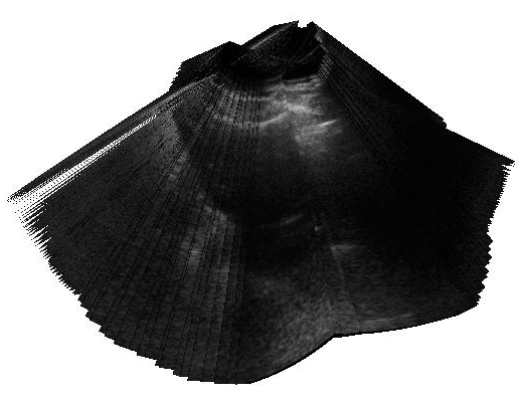

(a) $3 \mathrm{D}$ view

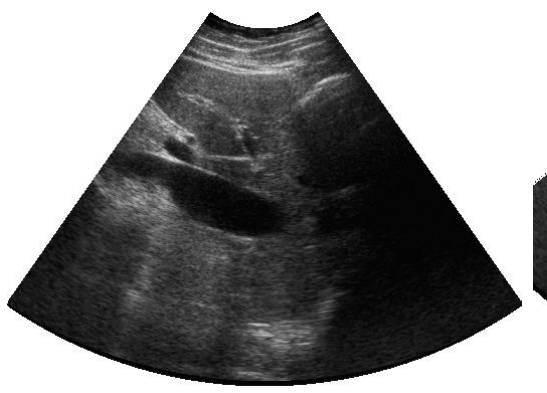

(b) ultrasound image

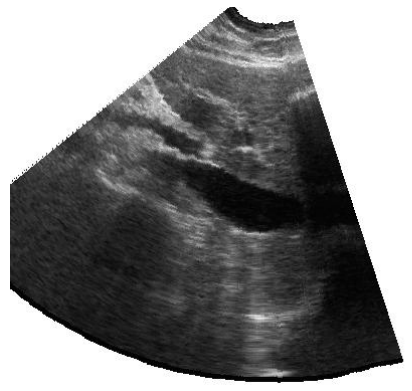

(c) reconstruction

Figure 5. Freehand ultrasound sweep of the human liver. NCC for the shown images is 0.60.

We repeated a particular calibration $(4 \mathrm{C} 1$ transducer at $18 \mathrm{~cm}$ depth) in-vivo on a human liver. The two required sweeps were combined to achieve a consistent data set within one breath-hold, and without removing the probe from the skin (see figure 5(a), angulated, twisted $90^{\circ}$, angulated back). The result deviates in the calibration parameters from the phantom-based results less than $2 \mathrm{~mm}$ and $4^{\circ}$, see table 1 (bottom). If the patient or volunteer steadily maintains the breath-hold and does not shift his position during the acquisition (10-20 seconds), the only additional error source with respect to phantom data arises from lower structures pulsating with the heart-beat (e.g. the portal vein).

\subsection{Temporal Calibration}

We achieved temporal calibration by performing a linear forward-backward motion on the phantom. Each 4 images in the forward motion were reconstructed from the backward motion, and vice versa. A one-dimensional Brent-line-optimization ${ }^{9}$ was run on the temporal delay parameter. It alters the spatial information of every recorded frame by interpolating between the neighboring tracking matrices, using a quaternion-based method. ${ }^{10}$ We consistently obtained a lag between the video-frames and tracking information of $100 \mathrm{~ms}$.

The linear motion used here makes the temporal calibration mostly independent of the spatial calibration parameters. For bootstrapping the calibration, it is sufficient to optimize the spatial calibration parameters with zero temporal lag, successively run the temporal calibration, and refine the spatial calibration with the temporal result. In our experience, the temporal delay is very similar for all different transducers and depth settings, and in fact only needs to be established once for a certain hardware setup.

\section{DISCUSSION}

We presented novel methods for spatial and temporal ultrasound calibration, that can be performed on any ultrasound phantom or in-vivo. We have expanded on the theoretical foundation of the method, and proved that the chosen acquisition geometry allows one to recover all spatial calibration parameters. Our result on a groundtruth 3D ultrasound machine, as well as freehand ultrasound calibration on both phantom and human liver data show that the method is reliable and precise. We believe it is a very convenient alternative to other methods published in the literature, that might especially be beneficial if the availability of specialized tools, phantoms and 3D imaging of those phantoms, is limited. As an advanced application, freehand ultrasound systems continuously recording during an exam or procedure could detect perpendicular image frames from analyzing the tracking trajectories, and automatically verify and correct some or all calibration parameters in the background. Besides, our algorithm might be used in conjunction with methods based on motion of successive ultrasound frames, ${ }^{7}$ to increase overall stability and accuracy by combining small- and large-scale anatomic clues.

\section{REFERENCES}

1. L. Mercier, T. Lang $\varnothing$, F. Lindseth, and D. Collins, "A review of calibration techniques for freehand 3-D ultrasound systems," Ultrasound in Med. ES Biol. 31, pp. 449-471, 2005.

2. R. Prager, R. Rohling, A. Gee, and L. Berman, "Rapid calibration for 3-D freehand ultrasound," Ultrasound in Med. E Biol. 24, pp. 855-869, 1998. 
3. F. Rousseau, P. Hellier, and C. Barillot, "Confhusius: A robust and fully automatic calibration method for 3D freehand ultrasound," Medical Image Analysis 9, Sept. 2005.

4. A. Khamene and F. Sauer, "A novel phantom-less spatial and temporal ultrasound calibration method," in MICCAI Proceedings, Oct. 2005.

5. D. Muratore and J. R.L. Galloway, "Beam calibration without a phantom for creating a 3-D freehand ultrasound system," Ultrasound in Med. E Biol. 27, pp. 1557-1566, 2001.

6. J. Blackall, D. Rueckert, C. M. Jr., G. Penney, D. Hill, and D. Hawkes, "An image registration approach to automated calibration for freehand 3D ultrasound," in MICCAI Proceedings, pp. 462-471, 2000.

7. E. Boctor, I. Iordachita, G. Fichtinger, and G. Hager, "Ultrasound self-calibration," in Proceedings of SPIE Medical Imaging, 6141, 2006.

8. W. Wein, F. Pache, B. Röper, and N. Navab, "Backward-warping ultrasound reconstruction for improving diagnostic value and registration," in MICCAI 2006 Proceedings, Lecture Notes in Computer Science, Springer, Oct. 2006.

9. W. Press, S. T. abd W.T. Vetterling, and B. Flannery, Numerical Recipes in C, Second Edition, CRC Press, Inc., 1992.

10. K. Shoemake, "Animating rotation with quaternion curves," in SIGGRAPH proceedings, 1985. 